Meta

Journal des traducteurs

Translators' Journal

\title{
La transa ou l'amour deux lalangues
}

\section{Betty Milan}

Volume 27, numéro 2, juin 1982

URI : https://id.erudit.org/iderudit/003650ar

DOI : https://doi.org/10.7202/003650ar

Aller au sommaire du numéro

Éditeur(s)

Les Presses de l'Université de Montréal

ISSN

0026-0452 (imprimé)

1492-1421 (numérique)

Découvrir la revue

Citer cet article

Milan, B. (1982). La transa ou l'amour deux lalangues. Meta, 27(2), 189-195.

https://doi.org/10.7202/003650ar

Ce document est protégé par la loi sur le droit d'auteur. L'utilisation des services d'Érudit (y compris la reproduction) est assujettie à sa politique d'utilisation que vous pouvez consulter en ligne.

https://apropos.erudit.org/fr/usagers/politique-dutilisation/ 


\section{LA TRANSA OU L'AMOUR DEUX LALANGUES}

BetTy Milan

Tudo se finge primeiro, germina autêntico é depois G. ROSA, Grande Sertao Veredas

On ne parle d'une langue que dans une autre langue. J. LACAN, Séminaire du 15.XI.1977.

Selon une certaine perspective, ancrée dans l'illusion du métalangage, la traduction serait la reformulation d'un même message dans un idiome différent, réussite ou échec s'appréciant en fonction de la plus ou moins grande permanence du contenu dans le passage de la langue de départ à la langue d'arrivée. L'accent est mis sur la «dénotation», «ce je veux dire qui dit toujours le ratage de l'intention dans le dire» ', le but étant alors la production d'un texte transparent par rapport à l'original.

À cette idée de la transparence qui se fonde sur la supposition d'une hétérogénéité entre la pensée et le langage, des langues en tant qu'actualisations particulières d'un signifié transcendental, Meschonnic ${ }^{2}$ a opposé la traduction comme réénonciation spécifique d'un sujet historique, interaction de deux poétiques ou, autrement dit, la traduction en tant que décentrement, contraire à ce qu'il nomme annexion, effacement du rapport entre les langues, illusion du naturel, comme si un texte en langue de départ était écrit en langue d'arrivée, abstraction faite des différences de culture et de structure linguistique. L'annexion est ainsi une procédure où ce n'est pas par la différence qu'il y aurait une transmission du même, mais par son effacement, la traduction fonctionnant ici comme là transmission de la psychanalyse dont le présupposé serait l'identification.

Dans la perspective du décentrement qui nous intéresse, le texte est abordé comme un poème, la traduction vise le style, et le message est supposé constitué par l'acte même de traduire, qui, de ce fait, n'est pas conçu comme une opération sur le langage, mais comme une opération de la langue, impliquant donc un sujet.

Dans cette perspective, il s'agit pour nous de cerner la spécificité de l'opération traduisante, en considérant d'une part le traducteur dans son rapport au texte et d'autre part la traduction elle-même, pour montrer que la transmission de la psychanalyse par la traduction suppose la production d'un nouvel

1. J.M. Ribettes: Noms du texte, à paraître.

2. J. Meschonnic: Propositions pour une Poétique de la traduction. 
indicible, et, s'agissant de Lacan, de l'ouverture dans une autre langue d'un espace de lisibilité illisible.

TRADUIRE, ANALYSER, ÉCRIRE

Ayant à faire un texte original, en vue d'un texte à produire, le traducteur attend que l'original reçoive d'une autre langue un sens, il attend suspendu à une certaine écoute, celle de ce qui du sens dans cette autre langue s'insinue dans ce qui est écrit.

Entre l'original et le texte cible, cette écoute qui en s'écrivant produira le nouveau texte ou, autrement dit, dans sa lecture de l'original le traducteur envoie à l'Autre (lieu, ici langue, d'où la parole prend son sens) un message, pour recevoir de celui-ci son propre message dans la langue cible, sous la forme d'une écoute qui s'écrit et ainsi fait du traducteur son sujet.

Rapportée à la subjectivité, la traduction demande à être distinguée de l'écriture d'une part, de l'acte analytique d'autre part, dont on dit qu'il est aussi traduction.

Écoute qui s'écrit, avons-nous énoncé pour la traduction. La même formule vaudrait pour l'écriture, si ce n'était qu'ayant à faire au texte de ce qui de son inconscient s'est inscrit, l'écrivain, lui, est suspendu à l'écoute d'un message qui lui vient directement de l'Autre, pour devenir le sujet d'une inscription inconsciente qui s'écrit.

Autrement dit, pour celui qui traduit: ce qui s'entend dans ce qui se lit. Pour celui qui écrit : ce qui s'entend dans ce qui est inscrit.

Quant à l'analysant, dans la mesure où il a affaire à un texte original, inconscient qui n'a pas encore été dit et qui insiste dans l'attente d'un dire, il doit dire, devenant ainsi sujet d'un dit dont ce qui est entendu s'inscrit. Il envoie un message à l'Autre, recevant de celui-ci son propre message, sous la forme d'une écoute qui s'inscrit.

Il y aurait donc entre l'analyse et la traduction, cette différence d'une écoute qui s'inscrit et d'une écoute qui s'écrit.

\section{UN SUJET EN MAL DE TRADUCTION ET EN MAL D'IMPOSSIBLE : L'HAINAMORATION}

Sujet d'une écoute qui s'écrit dans une autre langue et ne peut que s'écrire, le traducteur lit en fonction du texte à venir, le texte original étant par lui mis en dérive-dérivation du texte vers une autre langue. Cet $\grave{a}$ venir qui accroche le traducteur au texte, est ce sans quoi celui-ci n'aura pas d'effet là où le traducteur devra l'inscrire. Autrement dit, la traduction fait émerger le réel du texte, plaçant le traducteur dans la langue par laquelle il est concerné, langue qui fait de lui, dans son rapport à l'original, un lecteur en mal de traduction.

Dans la mesure où celle-ci ne peut que se faire, nous croyons pouvoir avancer qu'elle est l'effet d'un symptôme, au moyen duquel, le texte, objet $a$, se métonymise et la dérivation du sens se produit.

Le texte à traduire est tel que le traducteur s'y reconnaît en s'apercevant de son «étrangeté». Il est étranger à un texte auquel il appartient, et c'est pour ne point l'être qu'il traduit. 
Par ailleurs, il s'agit d'un texte supposé conférer au traducteur une reconnaissance par le savoir que celui-ci lui suppose, c'est-à-dire, que le texte doit légitimer le transfert du traducteur, qui lui demande de reconnaître sa supposition de savoir.

Écriture d'une lecture, la traduction est un glissement du sens qui se transfère d'une langue à une autre, pour que s'opère une transmission, dont le fantasme veut qu'il s'agisse du même et dont la réalité reconduit, au contraire, l'impossibilité du même entre deux langues, décalage qui fait du traducteur un lecteur confronté en permanence à l'impossible.

Pour autant que le métalangage n'existe pas, ce qui se transmet ne peut être que de l'ordre du sens, le traducteur étant par conséquent confronté à ce qui n'est pas intégralement transmissible, le réel du texte en tant qu'il lui résiste. Étant un lecteur supposé connaître une langue dans l'attente d'un savoir qui lui est donné ou refusé, le «ce n'est pas ça» précédant toujours le «c'est ça », moment où ça se trouve, nous pourrions dire que le traducteur est un lecteur en mal d'impossible.

En mal de traduction et en mal d'impossible, il entretient un rapport singulier au texte. Aimant un texte qui lui résiste puisque toute traduction suppose un indicible, le traducteur, sujet supposé savoir deux langues, ne fait qu'affronter son ignorance, l'impossibilité étant vécue comme impuissance et l'amour du texte donnant lieu à l'hainamoration. Le traducteur vit ainsi sa division entre deux langues à l'égard desquelles il se définit, imaginairement, par un savoir, réellement par un non-savoir. Sa division est donc telle qu'il y a une supposition de savoir concernant deux langues, et un non-savoir qui n'a pas de langue.

Face à cet impossible et à l'impuissance, la tâche du traducteur ne peut s'accomplir que par une symbolisation du réel de la division, c'est-à-dire, la division du sujet entre le signifiant d'une langue et son équivalent impossible dans une autre, division dont le présupposé est l'existence du métalangage. Autrement dit, la traduction ne devient possible que par une désimaginarisation de la langue, dissolution du fantasme de métalangage.

\section{L'AMOUR ENTRE DEUX LANGUES}

Qu'en est-il de cet impossible à traduire sinon précisément un irréductible indicible imposant ceci qu'entre deux langues il n'y a pas de rapport, il n'y a qu'un amour possible qui oblige à traiter le texte original comme un poème, la traduction qui le prendrait pour un message s'orientant à l'aide d'une herméneutique qui imaginariserait le texte.

Si le texte original fonctionne comme un poème, la traduction est la transmission d'un style dont résulte forcément la production d'un indicible dans la langue de départ.

A cet égard, Lacan nous dit: «Le style c'est l'homme, en rallierons-nous la formule à seulement la rallonger: l'homme à qui l'on s'adresse ${ }^{3}$ ?» «Nous voulons du parcours dont ces écrits sont les jalons et du style que leur adresse commande, amener le lecteur à une conséquence où il lui faille mettre du sien. »

3. J. Lacan (1966): Ecrits, Paris, Seuil, p. 9. 
Si celui à qui le texte s'adresse doit y mettre du sien, le propre du style est donc l'incomplétude dont résulte l'ambiguité du sens.

Or, la question serait de savoir ce qui pourrait être demandé par Lacan à une traduction: en quoi celle-ci serait-elle transmission de son style.

Orientée par une écriture qui trouve sa référence dans le discours, où l'après-coup est déterminant et l'expérience de l'incertitude un effet désiré, la traduction n'exige pas ici d'éviter l'équivoque, elle ne doit pas se laisser piéger par la recherche de la signification dernière, mais doit au contraire, dans la mesure où la résistance du texte est l'opération même du style, préserver les arêtes du texte, rouvrir dans une autre langue la possibilité d'une incertitude, créer la possibilité qu'un lecteur soit du nouveau texte un sujet.

Ce qui veut dire aussi un lecteur confronté à une certaine illisibilité, seule l'illisibilité donnant toujours à écrire et sans cesse à lire.

L'illisibilité du texte lacanien est telle que la lecture fait partie du texte, à la manière dont Freud découvre que les interprétations, les déformations et les oublis font aussi partie du rêve. Autrement dit, la transmission qui y est supposée, demande que le récepteur soit lui-même un émetteur et que la lecture soit ainsi une réécriture du texte.

Si dans cette dérivation du sens qu'est la traduction, c'est le style qui doit se transmettre, et si le style est cette valeur référentielle qui représente un sujet en marquant sa place d'énonciation, traduire c'est énoncer d'une position subjective, c'est trouver dans l'autre langue un même recoupement de celle-ci par le désir, produisant une même suspension du sens, une même ellipse ou béance pour actualiser ainsi un récepteur capable d'une même écoute.

Or, puisqu' «il n'y a pas plus de pragmatique du style que de description objective..., il n'y a pas non plus de théorie de l'élaboration structurale d'un style et de ses effets de sujet ${ }^{4} »$, puisqu'en somme il n'y a pas de stylistique prévisionnelle, comment, ce même recoupement de la langue par le désir, le trouver, sinon par une voie entièrement empirique, au sens précis de tenter, s'efforcer, essayer, voie dans laquelle chaque cas est unique, le savoir seulement par expérience et où le traducteur doit nécessairement supporter son opération par un non-savoir.

Ainsi, dans un transfert au texte, soutenu par une supposition de savoir et une demande de reconnaissance, le traducteur s'appuie imaginairement sur une connaissance qu'il doit suspendre, pour qu'il y ait tra-duction, trans-lation, trans-port d'une langue à une autre, ou, une transa ${ }^{5}$ entre deux langues.

Cette suspension, cette ignorance qui est le symptôme d'un savoir, est-ce par quoi, dans l'assomption progressive du symptôme, un transfert se dissoudra, dont l'effet réel est la transmission.

Maintenant je voudrais vous parler de ce à quoi l'on est confronté en traduisant Lacan en brésilien.

4. J.M. Ribettes: Noms du texte.

5. Mot d'argot passe-partout, dont le sens est imprécis renvoyant à entente, combinaison, accord, pacte, communication, liaison, trame, complot, machination. 
S'il est vrai d'une part qu'il faut traduire Lacan comme si on faisait des mots croisés, c'est-à-dire trouver le signifiant, d'autre part, il faut tenir compte de la signification, puisque tout en étant par son style, mise en scène de l'inconscient, le texte lacanien se réfêre au discours même de l'inconscient. Ici, ça parle et ça parle de ça. Ce texte qui a des effets poétiques ne peut pourtant pas se traduire comme un poème.

Ainsi, dans le texte de Lacan, quand il s'agit par exemple de «la lettre, l'être et l'autre", la traduction «A letra, o ser e o outro" qui rend compte exactement du signifié, implique, en faisant disparaître l'allitération, une perte de l'information signifiante. Ou encore, autre exemple, il peut s'agir d'un signifiant polysémique impliquant le choix d'un signifié : «le vol de la lettre», où les deux sens de vol n'existent en brésilien qu'à travers deux signifiants différents- roubol vôo da carta.

Si dans ces exemples, il existe un choix, dans d'autres cas la langue ne laisse au traducteur nul choix. C'est alors l'intraduisible, tel le ne explétif, dont vous connaissez l'importance dans la théorie pour ce qu'il en est du sujet de l'énonciation ou, tel encore, l'article partitif, ni l'un ni l'autre n'existant en brésilien.

À ces difficultés proprement linguistiques, d'autres s'ajoutent qui doivent nécessairement affronter la tradition psychanalytique dans la langue cible, la façon dont certains concepts ont été précédemment traduits.

Ainsi, par exemple, le ça, traduit en brésilien par $i d$, solution qu'il nous a fallu écarter dès la traduction des Écrits techniques de Freud. Le id est la solution de la Standard Edition qui l'a préférée au it, au nom du parallélisme avec l'ego, terme alors reçu depuis longtemps. Sans discuter précisément le mérite de la solution anglaise, adopter celle-ci dans la langue brésilienne ne se justifie pas. La façon même dont Freud justifie le choix du terme $E s$ nous l'indique : "Vous allez probablement vous opposer au fait que nous ayons choisi, pour désigner nos deux instances ou provinces psychiques, des mots simples au lieu de vocables grecs grandiloquents. Seulement nous aimons rester en contact avec la façon populaire de penser, dont nous préférons rendre les concepts utilisables scientifiquement, plutôt que de les rejeter. Il n'y a aucun mérite en cela : nous devons ainsi procéder, parce que notre savoir doit être entendu de nos malades, souvent très intelligents, mais pas toujours lettrés. Le Ça impersonnel s'associe immédiatement à certaines expressions de l'homme ordinaire. «Ça $m^{\prime}$ 'a traversé l'esprit», dit-on. «En moi il y avait ça, qui à ce moment-là était plus fort que moi ». «C'était plus fort que moi ». (G.W. XIV, p. 222. Traduction par J. Bollot, J. Ribettes, et moi-même). Freud nous montre ici que la théorie se doit d'utiliser le matériel signifiant de la langue dans laquelle elle s'élabore. Traduire un texte de Freud ou de Lacan, c'est faire exister la théorie psychanalytique dans une autre langue, c'est-à-dire, en faire exister les termes dans la langue où se traduit la théorie et où elle se fera entendre. $A$ cet égard, et même si dans les Écrits techniques de Freud, Lacan utilise alternativement le id et le ça, il vaut la peine de remarquer que dans les Séminaires et les écrits ultérieurs, le ça prévaudra définitivement. Il s'agissait donc pour traduire le ça, de trouver dans la langue brésilienne un terme qui prendrait simultanément en considération et la solution 
française et le $E s$ allemand, pronom neutre dont il n'y a d'équivalent grammatical exact ni en français ni en brésilien. Le Es a été introduit par Freud dans Das Ich und das Es, pour désigner les caractères psychiques autres que ceux du moi (G.W. XIII, 251). Dans ce texte, Freud attribue le terme à Grodeck et cite Nietzsche qui désignait par là «ce qu'il y a de non-personnel, et, pour ainsi dire, de nécessaire par nature dans l'être $(G . W$. , XIII, 251). Le concept freudien désigne donc le domaine psychique étranger au moi, en se référant à ce qui est non personnel. En tant que pronom, en effet, le Es est impersonnel. Outre sa valeur de démonstratif, le ça fonctionne comme substitut d'un pronom impersonnel ( $\mathrm{Ne}$ mets pas tes pieds sur la table, ça ne se fait pas). Considérant les pronoms démonstratifs en brésilien, isso est de la même façon que ça le plus indéterminé, fonctionnant également comme pronom impersonnel (Não ponha os pés na mesa, isso não se faz). Ce fait grammatical, plus l'accent mis par Freud sur l'usage du Es dans certaines expressions courantes où l'on retrouve ça et isso: "C'était plus fort que moi » Isso era mais forte do que eu; «C'est ça» $E$ isso. Ce sont ces considérations qui nous ont conduite à abandonner le $i d$ et à traduire le ça par $o$ isso.

Un problème du même ordre s'est posé pour la traduction du moi, équivalent au Ich, allemand, et que nous avons, contrairement à la tradition psychanalytique brésilienne, traduit par eu. Solution qui a résulté de deux sortes de considérations. D'une part, l'usage en brésilien du terme ego, latin, à refuser, pour les raisons déjà mentionnées, d'autre part, la différence très importante entre le français et le brésilien en ce qui concerne la première personne du singulier, le français ayant deux pronoms personnels, le je et le moi, le brésilien n'en ayant qu'un seul, à savoir, $o e u$. Comment donc traduire le moi, si, du point de vue de la grammaire eu est équivalent à je. Or, dès que nous passons de la grammaire à l'analyse de la fonction du pronom personnel dans l'énonciation, cette difficulté peut se résoudre. En effet, tandis que ni l'anglais ni le français n'emploient le verbe sans le pronom, le brésilien, lui, le fait, la première personne s'exprimant ici par la seule désinence verbale: je vois, se traduit par vejo comme l'espagnol d'ailleurs veo. Quand je dis eu vejo, c'est une redondance ou bien une emphase, la fonction du eu étant ici celle du moi (forme tonique du pronom personnel). Ainsi, je vois doit être traduit tout simplement par vejo et moi, je vois, par eu vejo. Pour cela, et encore par l'examen de certaines expressions françaises (moi-même : eu mesmo, moi seul : eu so, etc.) où le moi pourrait être traduit par $e u$, nous avons adopté cette solution nouvelle.

Si je vous ai fait état de problèmes concrets posés par la traduction, c'était aussi pour vous dire que traduire c'est valoriser autrement les signifiants de la langue-cible, et donc, forcément, créer en elle un espace de lisibilité illisible, ce qui est contraire à la position des éditeurs, qui, au nom de la soidisant intelligibilité, trouvent dans la traduction de Lacan, l'occasion de ce que $\mathrm{j}$ 'appellerai un renversement pédagogique de ses textes.

Cette illisibilité dont je vous parle, vaut pour la traduction des Écrits et aussi, d'une autre manière pour la traduction des Séminaires, où l'on a affaire à un texte écrit qui n'en est pas un, puisque ce qui se lit a originellement été dit, et que, le texte établi, pour rendre sensible le discours qui le 
soutient, nous confronte souvent à une écriture que seul le discours justifie. Or, les exigences de l'écriture n'étant pas celles du discours, nous aurons parfois dans la traduction des Séminaires, à traduire ce qui ne s'écrit pas.

Pour finir, je dirais que la traduction est le symptôme de celui qui, dans la trans-mission de la psychanalyse a à faire exister la théorie dans une autre langue, métalalangue par laquelle nous sommes quelques-uns au Brésil et en France à être concernés, ce travail ayant été élaboré à la suite de la traduction par moi-même d'un Séminaire de Lacan et d'un cartel du Colégio Freudiano do Rio de Janeiro, où, avec M.D. Magno, nous avons pu aborder la question de la traduction et, en particulier, ce qui en est du texte de Lacan. 